\title{
Negative Regulation of Immunoglobulin Kappa Light-Chain Gene Transcription by a Short Sequence Homologous to the Murine B1 Repetitive Element
}

\author{
KALLE SAKSELA AND DAVID BALTIMORE* \\ The Rockefeller University, 1230 York Avenue, New York, New York 10021
}

Received 21 December 1992/Returned for modification 22 February 1993/Accepted 25 March 1993

\begin{abstract}
B-cell-specific expression of the immunoglobulin kappa light-chain (Igк) gene is in part accomplished by negative regulatory influences. Here we describe a new negatively acting element (termed $\mathrm{kNE}$ ) immediately

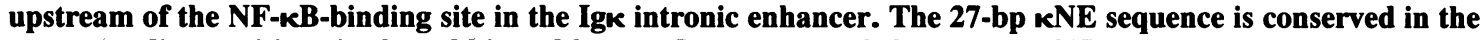
corresponding positions in the rabbit and human Igk genes, and the human $\kappa N E$ homolog was shown to have a similar negative regulatory activity. Data base searches using the mouse $\kappa N E$ sequence revealed a striking homology to murine B1 repetitive sequences. A sequence homologous to $\mathrm{kNE}$ and B1 was also noted in a previously identified silencer element in the murine T-cell receptor $\alpha$ locus. The homologous $T$-cell receptor $\alpha$ locus sequence, but notably not a corresponding 27-bp B1 consensus sequence, showed a negative regulatory potential similar to that of $\mathrm{kNE}$. The negative effect of $\mathrm{\kappa NE}$ by itself was not cell type specific but became so when paired with its $5^{\prime}$-flanking sequence in the Igא enhancer. A short (30-bp) fragment upstream of $\kappa \mathrm{NE}$ (termed $\kappa B S$ ) was found to be necessary and sufficient for abolishing the negative effect of $\kappa N E$ in $B$ cells. Point mutations in a T-rich motif within the $\kappa B S$ sequence allowed the transcriptional repression by $\kappa N E$ to be evident in B cells as well as other cells. As suggested by this cell-independent negative activity, proteins binding to the mouse and human $\mathrm{kNE}$ sequences were identified in all cell types tested.
\end{abstract}

The regulation of the immunoglobulin $\kappa$ light-chain (Igк) gene during B-cell development involves the Igא promoter and two distinct enhancer elements (for a review, see reference 11). The better characterized of the two Igk enhancers resides in the intron between the $J$ and $C$ regions, whereas the more recently identified $3^{\prime}$ enhancer is located several kilobases downstream of the Igא constant region. Both of these enhancers contain multiple sites for positive and negative regulation.

Some of the B-cell-specific activity of the Igk intronic enhancer can be attributed to the cell type specificity of the key positive regulator NF-kB (10), which becomes constitutively activated in the nuclei of maturing $\mathrm{B}$ cells (2). Although NF- $\mathrm{KB}$ can be activated from its inactive cytosolic form in most other cell types, this activation does not lead to an increased Igא intronic enhancer activity in non-B cells, such as $\mathrm{T}$ cells (17). Some of this cell type-specific negative regulation has been mapped to a 200-bp fragment upstream of the NF-kB-binding site (16). Removal of these sequences

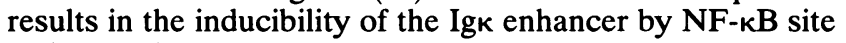
activators in non-B cells.

Here we show that another component of negative regulation at this locus can be ascribed to a short sequence immediately $5^{\prime}$ of the $\mathrm{kB}$ site in the Igk intronic enhancer. The activity of this element is not cell specific but becomes so when paired with a neighboring short sequence. The minimal negative element, but none of its flanking sequences in the Igא intronic enhancer, is closely related to the murine B1 repeat elements. Our experiments with a similar sequence from the T-cell receptor $\alpha$ locus (TCR $\alpha)$ enhancer indicate that B1-like sequences in other genetic contexts may be generally important as negative regulatory elements. Finally, we identify specific $\kappa \mathrm{NE}$-binding factors in nuclear

\footnotetext{
${ }^{*}$ Corresponding author.
}

extracts from several cells and show that this binding activity correlates with the transcriptional repression by $\mathrm{kNE}$.

\section{MATERIALS AND METHODS}

Plasmid constructs. The pfLUC plasmid was constructed by cloning the mouse fos promoter sequences $(-56$ to +109 ; from plasmid $\Delta 56$-fos-CAT; 6) to plasmid pBL-KS, made by inserting a new cloning site into the $\mathrm{pBL}$ vector (kindly provided by David Cobrinik, Whitehead Institute, Cambridge, Mass.) with the Photinus pyralis luciferase coding sequence in a pBluescript-based plasmid.

The NF-kB-driven plasmid pBIIX was constructed by inserting a synthetic fragment with two copies of the sequence ACA GAG GGG ACT TTC CGA GAG separated by four nucleotides (ATCT) in front of mouse fos promoter in plasmid pfLUC. The Igk fragments with different $5^{\prime}$ ends were polymerase chain reaction (PCR) amplified from an Igk $\mathrm{J}-\mathrm{C}$ region-containing plasmid and cloned into a SacI site immediately upstream of the first $\kappa B$-binding site in pBIIX. Thus, all the resulting inserts had identical $3^{\prime}$ ends with Igк sequence extending to position 3934 and had mutated residues at positions 3927 and 3929 (C to $G$ and $T$ to $G$, respectively) to create the $S a c I$ site (see Fig. 3A for sequence in this region). Since the functionally critical region of the Igк negative element was localized immediately $5^{\prime}$ of the introduced SacI site, we also constructed a plasmid in which the Igא insert shared its $5^{\prime}$ end with the 192-bp fragment but had a perfect wild-type sequence extending all the way to (and including) the $\kappa \mathrm{B}$ motif. No difference in the negative regulatory potential was observed between this construct and the SacI site-containing 192-bp Igk fragment construct. The plasmids carrying the 192-bp Igk fragment with point mutations $(192 \mathrm{~L}, 192 \mathrm{R}$, and $192 \mathrm{M})$ or deletions (192D) in the B1 homology region were constructed similarly but with longer 3' PCR primers including the corresponding 
base changes. The 27-bp kNE fragment-containing plasmid, as well as the other constructs with short inserted sequences used in the experiments shown in Fig. 2 and 3, were constructed by cloning corresponding synthetic doublestranded oligonucleotides into pBIIX or pfLUC. The mutant 96-bp Igk fragment (in construct 96B in Fig. 1A) was made by joining two partially overlapping PCR fragments (each having the desired point mutations in their overlapping 3' and $5^{\prime}$ ends) by a second PCR with the same primers used to amplify the 96-bp wild-type Igk fragment. All the constructs were verified by sequencing the region of the inserted fragment and the $\kappa B$ motifs.

Cells, transfections, and reporter expression assays. Approximately $5 \times 10^{6}$ cells were used for each transfection, which was done as described previously (7). The luciferase assay was performed $38 \mathrm{~h}$ after transfection using the Promega Luciferase Assay System. Phorbol 12-myristate 13-acetate (PMA), lipopolysaccharide, and forskolin used to stimulate the cells were purchased from Sigma. The HeLa, Jurkat, EL-4, BW5147, S194, P3X, PD31, 70Z/3, 22D6, and NFS- 5 cell lines and references for them can be found from the American Type Culture Collection catalog. The first two are human cell lines, whereas the rest are of murine origin. The efficiency of each transfection was monitored by a cotransfected cytomegalovirus enhancer-driven lac $Z$ construct kindly provided by Garry Nolan from our laboratory. The lysates used for luciferase assay were also tested for their $\beta$-galactosidase activity by using $o$-nitrophenyl- $\beta$-D-galactopyranoside (ONPG; Sigma) as a chromogenic substrate, and samples showing more than $25 \%$ variation in their optical densities at $420 \mathrm{~nm}$ were excluded.

Gel mobility shift assays. Nuclear extracts were prepared as previously described (3). The oligonucleotide probes were synthesized with an Applied Biosystems 392 DNA/RNA Synthesizer and their nucleotide sequences were as follows: mouse kNE, 5'-ACC TCT GTC ACC CAA GAG TTG GGA GCT CAG CTC CCA ACT CTT GGG TGA CAG AGG T-3'; mutant mouse kNE, 5'-ACC TCT GTT GTC CAA GAG TAC TGA GCT CAG CTC AGT ACT CTT GGA CAA CAG AGG T-3' (mutations in the sense-strand region of the oligonucleotide underlined); and human kNE, 5'-TGC TCT CCC ACC CAA CGG GTG GGA GCT CAG CTC CCA CCC GTT GGG TGG GAG AGC A-3'. The oligonucleotides were purified from an acrylamide gel, $100 \mathrm{ng}$ of each oligonucleotide was end labeled, extracted twice with phenol-chloroform, diluted to $2 \mathrm{ml}$ with TE (Tris-EDTA), boiled for $3 \mathrm{~min}$, and allowed to sit at room temperature to allow self-annealing. The labeled probes were concentrated and separated from unincorporated radioactivity by three rounds of microconcentration using Centricon-10 columns (Amicon). Nuclear extract $(10 \mu \mathrm{g})$ was incubated for $40 \mathrm{~min}$ at room temperature with $0.5 \mathrm{ng}$ of probe (corresponding to $3 \times 10^{5}$ $\mathrm{cpm}$ ) in a solution containing $10 \mathrm{mM}$ Tris (pH 7.5), $50 \mathrm{mM}$ $\mathrm{NaCl}, 5 \%$ glycerol, $1 \mathrm{mM}$ EDTA, and $1 \mathrm{mM}$ dithiothreitol together with $2 \mu \mathrm{g}$ of poly $(\mathrm{dI} \cdot \mathrm{dC})$ and $200 \mathrm{ng}$ of an unrelated short double-stranded oligonucleotide in a final reaction volume of $20 \mu \mathrm{l}$. In some reaction mixtures, $50 \mathrm{ng}$ of a specific competing self-annealed oligonucleotide was also added. The sequences of the TCR $\alpha$ and consensus B1 competitor fragments are given in Fig. 2B. The complexes were electrophoresed in gels containing $4 \%$ acrylamide, $0.25 \times \mathrm{TBE}$ (Tris-borate-EDTA) and $10 \%$ glycerol at $4^{\circ} \mathrm{C}$ for $7 \mathrm{~h}$ at $10 \mathrm{~V} / \mathrm{cm}$.

\section{RESULTS}

Characterization of the Igא negative element. To analyze the negative regulatory region in the Igא intronic enhancer, a 192-bp fragment upstream of the $\mathrm{kB}$ site was cloned into a luciferase reporter plasmid (pBIIX) in which transcription was stimulated by two copies of the murine Igk NF-kBbinding site in front of a minimal fos promoter (see Materials and Methods). This 192-bp fragment included most of the Igk intronic sequences used in a previous study (16) but extended closer to the $\mathrm{KB}$ site at the $3^{\prime}$ terminus. Other modifications to the previous study included the use of a smaller promoter to amplify the importance of the enhancer, and addition of an upstream polyadenylation site to block any read-through transcription initiated from the vector sequences.

We subjected this 192-bp Igk fragment to a series of deletions and point mutations (Fig. 1A). Luciferase activity of these constructs was measured after transient transfection into cells representing B-cell and non-B-cell lineages. In all experiments, a cotransfected heterologous reporter construct was used as an internal control for transfection efficiency and cell viability (see Materials and Methods).

After 4-h stimulation with $100 \mathrm{nM}$ PMA or $2 \mu \mathrm{g}$ of phytohemagglutinin per $\mathrm{ml}$, agents known to induce NF-kB activity, the vector containing two copies of the NF-кBbinding site showed strong activation in $T$ cells (Jurkat and EL-4), and in nonlymphoid cells (HeLa) (Fig. 1A and data not shown). In contrast, the luciferase activity produced by a corresponding construct lacking the $\mathrm{\kappa B}$ sites and having only the minimal mouse fos promoter (pfLUC) was less than $5 \%$ of this activity, indicating that we are indeed measuring transcription stimulated via the NF-kB-binding sites. As expected, high levels of luciferase activity were seen in S194 plasmacytoma cells transfected with pBIIX without the addition of exogenous NF- $\mathrm{kB}$ activators.

When the 192-bp Ig $\kappa$ fragment was introduced upstream of the two $\mathrm{kB}$ sites in the pBIIX vector, we found that the PMA-stimulated luciferase expression of non-B cells transfected with this construct was reduced to one-third of that of cells transfected with the parental vector pBIIX (shown in Fig. 1A for Jurkat cells). In contrast, in mature B cells (S194 or P3X), the 192-bp Igk fragment had a marginal or no effect on NF-kB-driven transcription. Also, no negative effect on PMA-stimulated luciferase expression by the 192-bp Igk fragment was observed in any pre-B-cell lines tested (22D6, PD31, 70Z/3, and NFS-5); instead, slightly higher luciferase activities were observed than in pBIIX-transfected cells (22D6 cells [Fig. 1A]).

Analysis of the effect of successive $5^{\prime}$ deletions of this 192-bp fragment indicated that its negative effect could be restricted to a 27-bp sequence (referred to as $\mathrm{kNE}$ ) immediately upstream of the NF-kB-binding site in the Igk intronic enhancer (Fig. 1A; see also Fig. 3A). When an even shorter 17-bp fragment was tested, the negative effect was weaker, but this construct still showed consistently $-50 \%$ lower transcriptional activity than the pBIIX construct. A multimer consisting of three copies of this 17-bp sequence had a negative effect slightly stronger than any of the monomeric Igk fragments (construct $3 \times 17$ in Fig. 1A).

We further examined the $\mathrm{kNE}$ sequence requirements by introducing mutations into the 192-bp Igk fragment within the 17-bp region indicated above (Fig. 1B). We found that the negative effect of the 192-bp fragment could be severely compromised by mutating only four (192L) or three (192R) nucleotides at either end of this 17-bp semipalindromic 


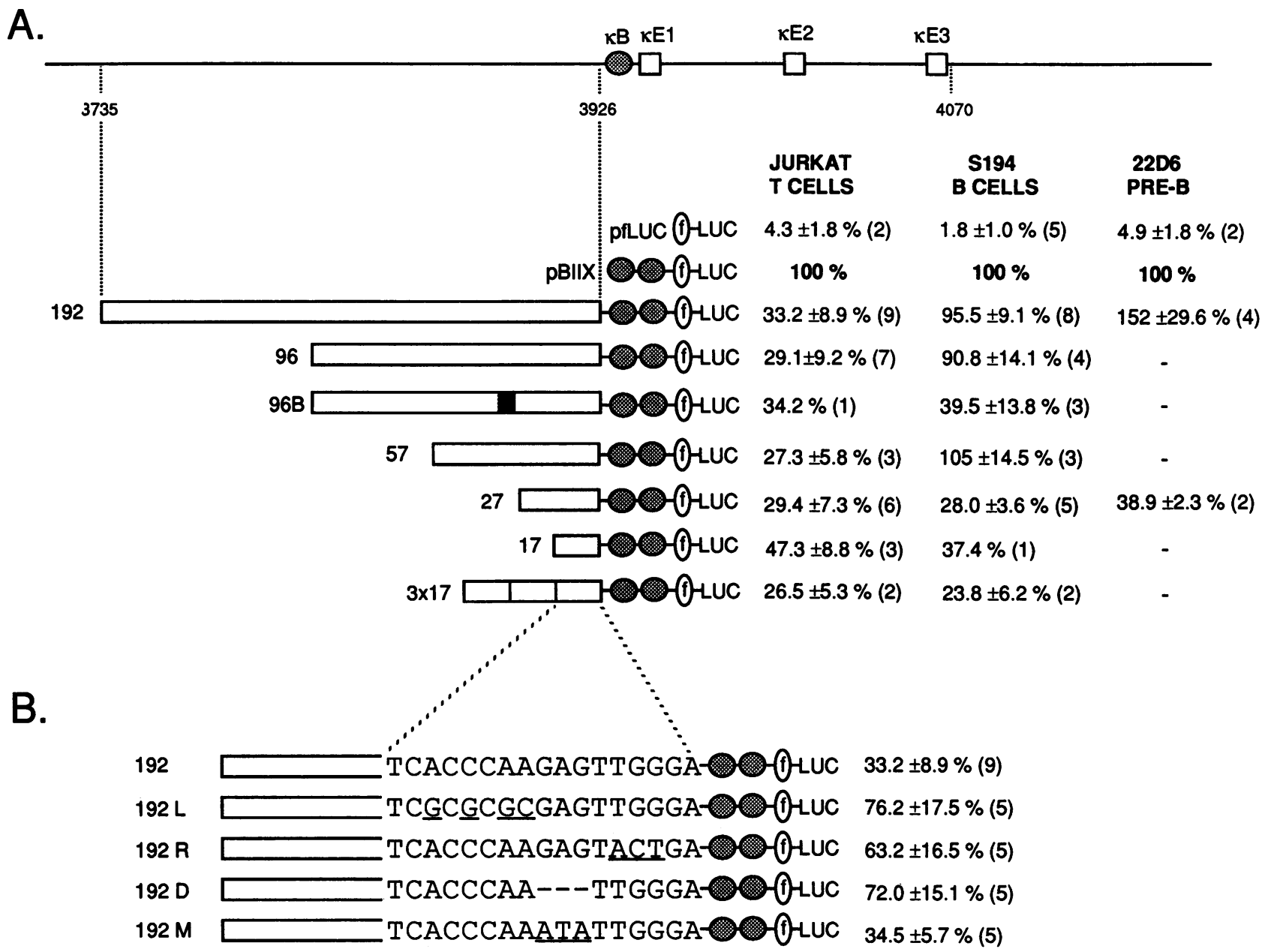

FIG. 1. Mutational analysis of a cell type-specific negative element in the Igk intronic enhancer. (A) The locations of the subcloned 192-bp fragment upstream of the NF-kB-binding site and the E-boxes within the murine Igk enhancer are illustrated schematically at the top of the figure. Different Igk fragments from this region were tested for their ability to downregulate transcription stimulated by two copies of the NF-kB-binding site (shaded ovals) that directed transcription from a minimal (-56 to 109) fos promoter (f) in plasmid pBIIX. The 192-bp Igk fragment and different $5^{\prime}$ truncated versions of this fragment were cloned $5^{\prime}$ of two $\mathrm{kB}$ sites in plasmid pBIIX. The lengths of the truncated Igk fragments that all share a common $3^{\prime}$ end (Igא residue 3926) are indicated to the left of each vector. Vector 96B is a mutated version of construct 96 and contains the GCGCGC sequence instead of TTCGTT in the position indicated by the dark box. Luciferase expression was then measured in PMA-stimulated T cells (Jurkat) and pre-B cells (22D6) as well as in unstimulated B cells (S194) transiently transfected with these vectors. In all experiments, the luciferase activity produced by the parental pBIIX construct was normalized to $100 \%$, and the activities of the other constructs ( \pm standard deviation) were expressed relative to this. The number of independent experiments performed with each construct is indicated in the parentheses. (B) Effects of point mutations in the 3' region of the 192-bp Igk fragment on transcriptional repression in PMA-stimulated Jurkat cells. The nucleotide sequence of the parental 192 construct within the indicated 17-bp region is shown on the top line. The specific nucleotide substitutions in the derived constructs 192L, 192R, and 192M are underlined, and the deleted nucleotides in the 192D construct are indicated by dashes. Luciferase expression of these vectors is indicated relative to that of the pBIIX vector, as described above. LUC, luciferase.

sequence, confirming the functional importance of this region, as indicated by our deletion studies. The negative effect of the 192-bp fragment was also affected by deletion of the three nucleotides, GAG, in the middle of this region (192D), but not by changing these residues to ATA (192M), suggesting that not every residue within this 17 -bp region is critical as long as a correct nucleotide spacing is conserved. Some residual negative effect remained in all of these 192-bp fragments, as was the case when the whole 17-bp region was changed to an unrelated sequence (not shown), indicating some contribution of the more 5 ' sequences, presumably the CT-rich region present in the 27-bp fragment but not in the 17-bp fragment shown in Fig. 1A.
Pierce et al. (16) defined a broadly localized negative effect in the Igк enhancer region upstream of the $\kappa B$ site. In our constructs, a negative activity is distinctly localized to a region that was truncated and therefore probably not active in the fragment studied by Pierce et al. (16). Conversely, we saw little or no regulatory activity in the Igk region upstream of the $\mathrm{kNE}$. The negative effect that we have mapped is also position dependent, because this activity was completely abolished by a 260-bp nonspecific pUC-derived fragment inserted between the $\mathrm{\kappa NE}$ - and NF- $\mathrm{kB}$-binding sites (not shown). We are uncertain why the activity defined in the earlier experiments was not evident in the present experimental set-up, but we have gone on to define the new activity 
A. $\begin{array}{ll}\text { Mouse } & \text { TACTĂ̈CTCTGTCACCCAAGAGTTGGCA } \\ \text { Human } & \text { TACTGCTCTCCCACCCAACGGGTGGA } \\ \text { Rabbit } & \text { CGATGCTCTCCCACCCTAGGCCTGACT }\end{array}$

B.

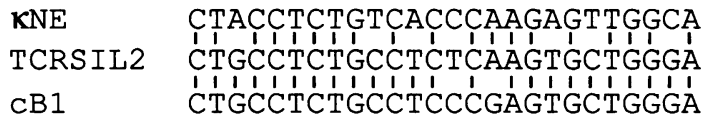

C.

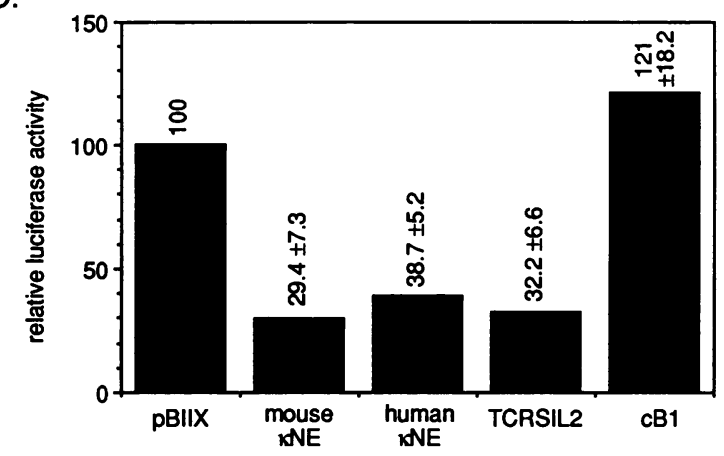

FIG. 2. Comparative analysis of sequences homologous to the murine $\mathrm{kNE}$. (A) Alignment of the corresponding human and rabbit Igk sequences with the 27-bp murine $\kappa N E$. The nucleotide residues conserved in all three species are shown in boldface type. (B) Comparison of the murine $\mathrm{kNE}$ and TCRSIL2 fragments and a murine B1 element consensus (cB1) sequences. (C) Analysis of transcriptional effects of the mouse $\mathrm{kNE}$ and other B1-homologous sequences. Twenty-seven-base-pair fragments corresponding to mouse and human $\mathrm{kNE}$, mouse TCRSIL2, and the consensus B1 element shown in Fig. 2B were introduced upstream of the $\kappa B$ sites in plasmid pBIIX as in Fig. 1A and tested for their effect on NF-kB-stimulated luciferase expression in PMA-stimulated Jurkat cells (human and mouse $\mathrm{kNE}$ ) or unstimulated S194 cells (TCRSIL2 and $\mathrm{cB} 1$ ).

because it has properties that suggest it has general significance.

To study whether the negative effect of the $\mathrm{kNE}$ was restricted to NF- $\mathrm{kB}$, we precisely replaced the $\kappa \mathrm{B}$ sites in the pBIIX construct and its derivative carrying the 192-bp Igא fragment (Fig. 1A) by two copies of the cyclic AMP responsive element consensus sequence (TGACGTCA [19]) and measured the forskolin-stimulated and unstimulated luciferase expression of these constructs in Jurkat cells. In both cases, an approximately threefold-lower luciferase activity was produced by the 192-bp Igא fragment-containing vector (not shown). Thus, the negative activity of $\mathrm{kNE}$ is not restricted to NF-kB, but it can downregulate transcription activated via other binding sites.

A sequence homologous to the murine $\mathrm{kNE}$ is also present adjacent to the $\kappa B$ site in the human Ig $\kappa$ intronic enhancer (Fig. 2A), suggesting that it might have a similar negative regulatory role. We tested this directly by introducing the human 27-bp kNE sequence into the pBIIX reporter construct and found that this element could indeed interfere with NF-kB-driven transcription and have a negative effect corresponding to that of the mouse $\mathrm{kNE}$ (Fig. $2 \mathrm{C}$ ). The conservation of $\mathrm{kNE}$ between the mouse, human, and rabbit sequences highlights the significance of this element and points to the importance of the specific residues indicated by our mutagenesis studies.

KNE is homologous to the B1 repeat element. When we screened the GenBank data base using the 27-bp murine kNE sequence, a large number of almost perfect alignments was found. This turned out to be due to the homology of kNE to the mouse B1 short repetitive sequences and their primate Alu counterparts (Fig. 2B). This homology was shared only by the $\mathrm{kNE}$ region and did not extend to its flanking sequences in the Igk enhancer.

Interestingly, we noted that a B1-homologous sequence corresponding to $\mathrm{kNE}$ is also present in a previously characterized silencer element, TCRSIL2 (24), in the mouse T-cell receptor $\alpha$ gene locus (Fig. 2B). We tested this 27-bp $\mathrm{TCR} \alpha$ fragment in our $\mathrm{kB}$-driven reporter gene system and found it to have a negative transcriptional effect similar to that of $\mathrm{kNE}$ (Fig. 2C). The plasmid constructs used in these experiments had the TCR $\alpha$ sequence inserted in an orientation opposite to that of $\mathrm{kNE}$ (which was in the sense orientation). Both TCR $\alpha$ and Igk 27-bp fragments were, however, able to repress transcription in either orientation.

We then tested a 27-bp B1 consensus fragment ( $\mathrm{cB1}$ in Fig. 2B) corresponding to the single most prevalent sequence in this region of B1 elements (on the basis of our GenBank data base searches and references 12 and 18). However, no negative effect on NF-kB-stimulated transcription was observed with this consensus B1 fragment, suggesting that not all $\mathrm{B} 1$ repeats have negative regulatory potential. The data shown in Fig. 2C on the transcriptional effects of the human and mouse 27-bp kNE fragments were obtained from PMAstimulated Jurkat cells, and the data on the 27-bp TCR $\alpha$ or consensus B1 fragments were obtained from S194 cells. However, as shown for the mouse $\mathrm{kNE}$ in Fig. 1A, none of these short B1-homologous fragments showed cell type specificity in their action, and the negative effects of the mouse TCR $\alpha$ fragment and human $\mathrm{KNE}$ were equally evident in T cells and B cells (data not shown).

Inactivation of $\mathrm{kNE}$ in $B$ cells by an upstream element. When the series of constructs containing differentially $5^{\prime}$ deleted versions of the 192-Igk fragment were tested in S194 cells (Fig. 1A), we found that disruption of the $\mathrm{kNE}$ sequence from its upstream flanking region resulted in an activation of its negative potential in B cells (Fig. 1A). In contrast to the 192-bp Igk fragment, the 27-bp kNE fragment could repress transcription in all cell types tested, including B cells. The presence of only $30 \mathrm{bp}$ of Igk sequences upstream of the $\mathrm{kNE}$, however, was sufficient to restore the cell type specificity of the $\mathrm{kNE}$, since no negative effect was observed for the 57-bp fragment when tested in S194 cells (Fig. 1A). Therefore, this 30-bp region is a critical determinant of the cell type specificity of $\mathrm{kNE}$. The nucleotide sequence of this 30-bp fragment (referred to as $\mathrm{kBS}$ for Igk B-cell specificity) is shown in Fig. 3A. The $\kappa B S$ sequence contains two copies of the T-rich motif GTTTT separated by a $\mathrm{C}$ residue. Disruption of this region of the $\mathrm{kBS}$ element in the reporter construct with a 96-bp Igk fragment by a TTCGTT-to-GCGCGC mutation activated the negative potential of this fragment in S194 cells, rendering it as potent a negative element in B cells as in non-B cells (compare constructs 96 and 96B in Fig. 1A).

To test whether the $\mathrm{kBS}$-mediated B-cell-specific alleviation of the negative regulation by $\mathrm{kNE}$ could be due to an independent positive transcriptional effect by $\mathrm{kBS}$, we introduced two copies of the $\mathrm{kBS}$ sequence in front of the minimal fos promoter in the pfLUC luciferase construct. Luciferase expression of the resulting plasmid pкBS was 
A.

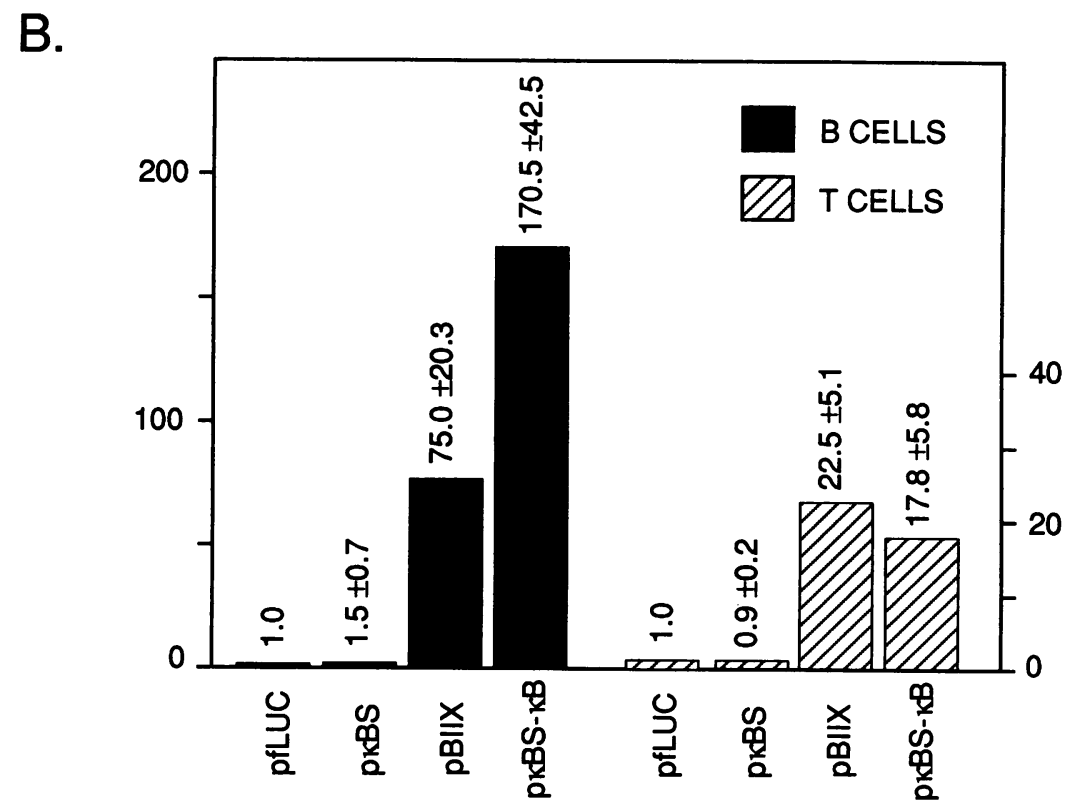

FIG. 3. Cell-type-specific enhancement of transcriptional activation by NF- $\kappa B$ via a T-rich sequence ( $\kappa B S$ ) from the Igk intronic enhancer. (A) Nucleotide sequence of the murine Igк gene from positions 3880 to 3952 . The bars indicate the exact sequences of the $\kappa B S$ and $\kappa B$ motifs introduced in the fos promoter-luciferase reporter constructs. The 27-bp $\mathrm{kNE}$ sequence is also indicated to illustrate the close proximity of

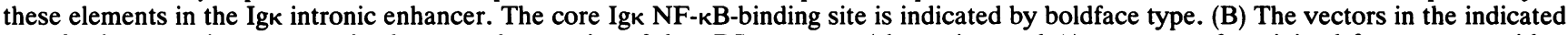
transfection experiments contained two tandem copies of the $\kappa B S$ sequence (shown in panel A) upstream of a minimal fos promoter either

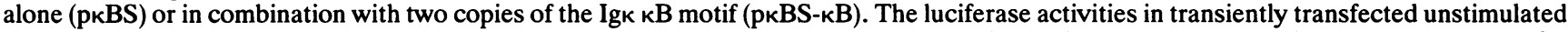
B cells (S194) cells or PMA-stimulated T cells (Jurkat) are expressed relative to the activity of a construct containing only the minimal fos promoter (pfLUC). Separate scales for the relative transcriptional activity are given for the two cell lines. The mean values from three or more independent experiments and their standard deviations are shown.

then compared with that of pfLUC in B cells and non-B cells. No significant transcriptional stimulation was observed in either cell type, suggesting that the $\kappa B S$ element by itself has no transcriptional activation potential.

However, when the two copies of the $\mathrm{kBS}$ sequence were cloned $5^{\prime}$ of the NF- $\mathrm{NB}$ binding sites in plasmid pBIIX (resulting in plasmid pкBS-кB), a marked enhancement in transcription was observed in B cells (Fig. 3B). The strong transcriptional activation by two murine Igк кB sites in S194 cells was further increased two- to threefold by the additional tandem $\kappa B S$ element. In contrast, no such potentiation of the NF- $\mathrm{kB}$-activated transcription by $\kappa \mathrm{BS}$ was seen in PMA-stimulated Jurkat cells; instead, less luciferase was expressed in these cells by the pкBS-кB plasmid than by pBIIX (Fig. 3B). A sequence homologous to the mouse $\kappa B S$ element is also present adjacent to the $\mathrm{kNE}$ element in the human kappa enhancer. A similar B-cell-specific enhancement of transcriptional activation via the Igk $\kappa B$ site was also observed with the human $\mathrm{kBS}$ sequence (data not shown).

These data suggest a role for the $\mathrm{kBS}$ element as a cell type-specific modulator of transcriptional activation via the NF-kB-binding site within the Igk intronic enhancer. Thus, combined influences by these three closely positioned binding sites ( $\kappa \mathrm{BS}, \kappa \mathrm{NE}$, and $\kappa \mathrm{B})$ appears to create a composite enhancer element whose activity is regulated by NF-кB but is mainly restricted to the B-cell lineage.

Sequence-specific nuclear factor binding to $\mathrm{kNE}$ correlates with its negative regulatory potential. To characterize factors mediating the negative transcriptional effect of the $\mathrm{kNE}$, we prepared nuclear extracts from various B-cell and non-B-cell lines and tested them for specific binding to the $\mathrm{kNE}$ sequence in gel retardation assays. In agreement with the ubiquitous activity of $\kappa \mathrm{NE}$, a specific complex binding to the mouse $\kappa$ NE probe was detected in mouse (S194 and 22D6) and human (Jurkat and HeLa) cell lines regardless of their lineage of differentiation (complex $A$ in the left panel in Fig. 4; also data not shown). The specificity of DNA binding by complex A was strongly suggested by the experiments utilizing different unlabeled competitor binding sites and a mutated version of the $\mathrm{kNE}$ probe (discussed below). The binding of complex A was not very strong; this binding became evident only when glycerol was added to the mobility shift gels to stabilize the binding and increased when electrophoresis was carried out at $4^{\circ} \mathrm{C}$. Under these conditions, an additional slower migrating specific complex was also observed in HeLa cells ( $A^{\prime}$ in Fig. 4, left panel). This complex was not detected in nuclear extracts from other human or mouse cell lines. Complex $\mathrm{A}^{\prime}$ could therefore be 


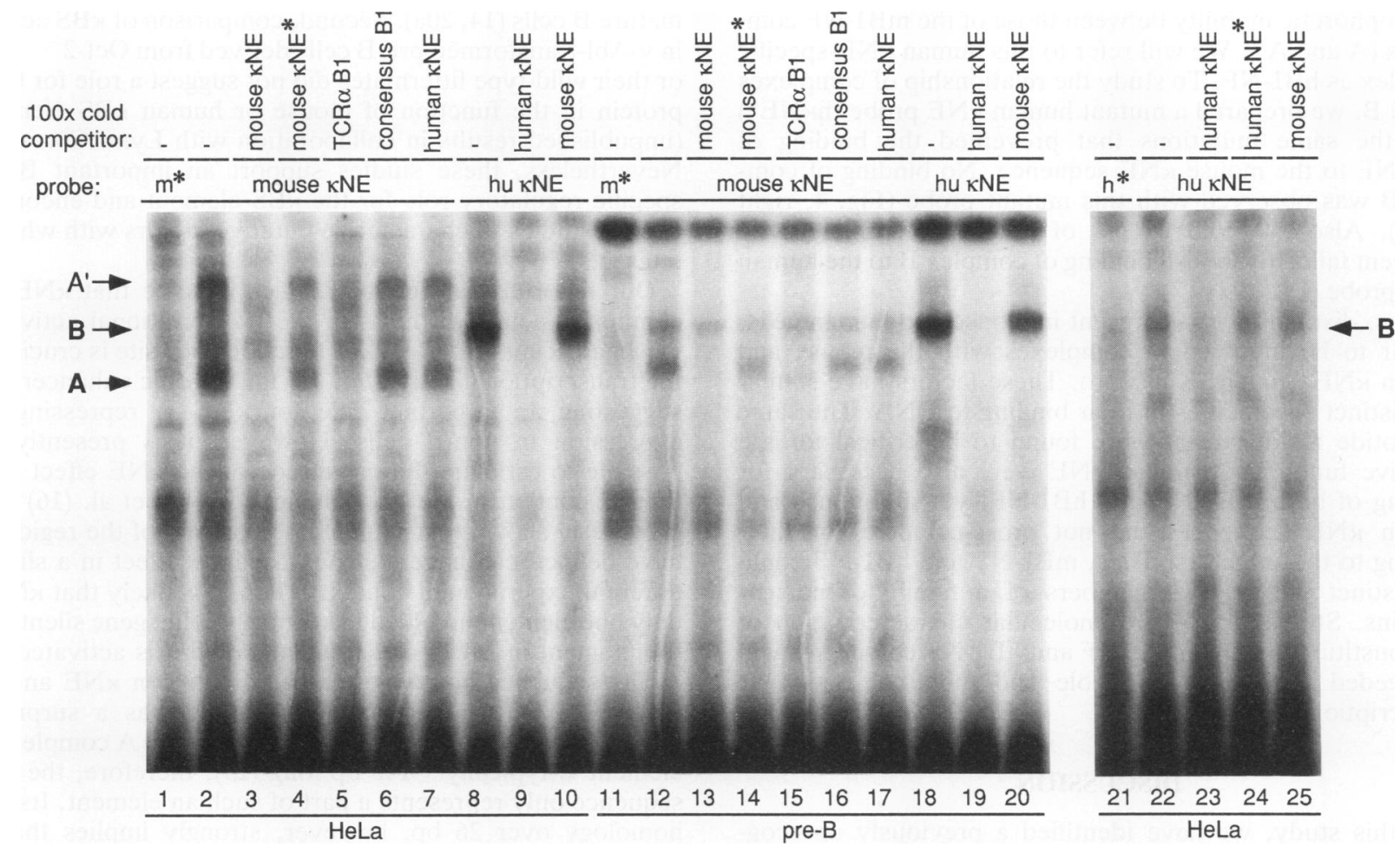

FIG. 4. Specific binding of nuclear factors to the mouse and human $\mathrm{kNE}$ sequences. Nuclear extracts from cells representing non-B (HeLa [human carcinoma cell line]) and B-cell lineages (22D6; $v$-Abl-transformed mouse pre-B-cell line) were tested for factors that could specifically bind to mouse and human (hu) $\mathrm{kNE}$ probes, as well as a mutant mouse and human $\mathrm{kNE}$ probes $(*)$ with changes in nucleotide residues critical for transcriptional repression by the murine $\mathrm{kNE}$ sequence. In some lanes, a 100 -fold excess of the indicated unlabeled competitor was also included in the binding reaction mixture. In lanes labeled -, no specific competitor was used. The details of the assay and the sequences of the oligonucleotide probes used are specified in the Materials and Methods. Complexes specifically interacting with the mouse (A and $\left.\mathrm{A}^{\prime}\right)$ and human (B) $\mathrm{kNE}$ sequences are indicated by arrows.

due to a cell-type-restricted $\kappa N E$-binding factor but more likely reflects a higher quality of the nuclear extracts prepared from HeLa cells. Although there is no direct evidence that the constituents of complexes $\mathrm{A}$ and $\mathrm{A}^{\prime}$ are related, we will refer to both of them as mB1-NF (for murine B1-binding nuclear factor).

To test whether binding of mB1-NF to the 27-bp kNE element correlated with its transcriptional repression potential, we prepared a mutant probe (mouse $\mathrm{kNE}^{*}$ [lanes 1 and 11 in Fig. 4]) in which residues CAC and TGG were changed to TGT and ACT, respectively (for details, see Materials and Methods). On the basis of our mutagenesis studies (Fig. 1B), these changes should abolish transcriptional repression by mouse $\kappa \mathrm{NE}$ and, therefore, presumably disrupt binding of any functionally relevant negative factors. No $\mathrm{mB} 1-\mathrm{NF}$ binding was observed with this mutant version of the $\mathrm{kNE}$ sequence, indicated by the complete absence of complexes $A$ and $A^{\prime}$ in Fig. 4 (compare lanes 1 and 2 and lanes 11 and 12). Identical arrays of complexes binding nonspecifically to both the mutant and wild-type probe highlighted the specificity of the loss of mB1-NF interaction with the mutant $\mathrm{kNE}$ probe. In agreement with these results, a 100 -fold excess of the mutant $\mathrm{kNE}$ fragment was unable to abolish B1-NF binding to the wild-type $\kappa \mathrm{NE}$ probe. In contrast, under the same conditions, a 100-fold excess of the wild-type $\mathrm{kNE}$ competitor completely abolished the B1-NF complex (Fig. 4). These data confirm that the mB1-NF complex binds specifically to the mouse $\mathrm{kNE}$ sequence and show that this interaction is dependent on the nucleotide residues critical for the negative regulatory potential of $\mathrm{kNE}$.

These results prompted us to use other fragments homologous to $\mathrm{kNE}$ and $\mathrm{Bl}$ and previously tested for their transcriptional regulatory potential as specific competitors for $\mathrm{B} 1-\mathrm{NF}$ binding to the mouse $\kappa \mathrm{NE}$ probe. It is striking that the $\kappa N E$-like fragment from the TCR $\alpha$ silencer, which had a negative regulatory potential comparable to that of $\kappa \mathrm{NE}$, was also able to efficiently abolish mB1-NF binding to the кNE probe (Fig. 4, lanes 5 and 15). In contrast, despite its close sequence similarity, the corresponding 27-bp mouse B1 repeat consensus fragment failed to abolish mB1-NF binding (lanes 6 and 16), which correlated with its lack of negative regulatory potential in reporter gene assays (Fig. 2C). These results substantiate the significance of the $\mathrm{mB} 1-\mathrm{NF}$ as a mediator of the negative regulation by $\mathrm{kNE}$ and suggest a role for it in negative regulation by a subset of other B1-homologous sequences dispersed in the mouse genome, such as the B1 homology region in the TCR $\alpha$ silencer.

The negatively acting human $\mathrm{kNE}$ homolog, however, did not abolish binding of $\mathrm{mB} 1-\mathrm{NF}$ to the mouse $\mathrm{\kappa NE}$ probe in nuclear extracts either from mouse or human cell lines (Fig. 4, lanes 7 and 17). In order to understand this finding, we prepared a similar mobility shift probe corresponding to the human $\kappa N E$ sequence. A single specific binding complex was observed by using the human $\mathrm{kNE}$ probe in mouse and human cell lines (complex B in Fig. 4), which had an 
electrophoretic mobility between those of the mB1-NF complexes $\left(A\right.$ and $\left.A^{\prime}\right)$. We will refer to this human $\kappa N E$-specific complex as hB1-NF. To study the relationship of complexes $A$ and $B$, we prepared a mutant human $\kappa N E$ probe (hкNE*) with the same mutations that prevented the binding of $\mathrm{mB} 1-\mathrm{NF}$ to the mouse $\mathrm{kNE}$ sequence. No binding of complex B was observed with this mutant probe (Fig. 4, right panel). Also, 100-fold excess of unlabeled human $\mathrm{kNE}^{*}$ fragment failed to abolish binding of complex $\mathrm{B}$ to the human KNE probe.

Thus, distinct factors, present in mouse and human cells, appear to be involved in complexes with the mouse and human $\kappa N E$ sequences in vitro. These factors have similar but distinct specificities in their binding to DNA. The same nucleotide residues that were found to be critical for the negative function of mouse $\mathrm{kNE}$ were also necessary for binding of both mB1-NF and hB1-NF, yet the mouse and human $\kappa \mathrm{NE}$ sequences did not cross-compete for their binding to these factors. Thus, mB1-NF and hB1-NF could be distinct but related members of a family of nuclear proteins. Studies examining molecular characterization of the constituents of the mB1-NF and hB1-NF complexes will be needed to clarify their role and mode of action in transcriptional repression.

\section{DISCUSSION}

In this study, we have identified a previously unrecognized short negative element ( $\kappa \mathrm{NE}$ ) close to the NF-kBbinding site in the Igk intronic enhancer. Computer analysis of the $\mathrm{kNE}$ sequence revealed a striking homology to the murine $\mathrm{B} 1$ repetitive element of the short interspersed repetitive element superfamily (see below). Nuclear factors specifically binding to $\kappa$ NE were identified and termed B1-NF. Functional significance of the B1-NF complex was supported by mutagenesis and binding site competition studies which established a strong correlation between B1-NF binding to $\mathrm{kNE}$ in vitro and transcriptional repression in vivo. Further characterization of B1-NF will be necessary to uncover the molecular mechanisms involved in the transcriptional repression by $\mathrm{kNE}$ and other similar elements.

The cell type specificity of $\kappa \mathrm{NE}$ was found to be determined by a short element, termed $\kappa B S$, immediately upstream of $\kappa \mathrm{NE}$ in the Igk enhancer. Disruption of the $\kappa \mathrm{BS}$ sequence allowed transcriptional repression by $\kappa \mathrm{NE}$ to be evident in $B$ cells. When analyzed separately from $\mathrm{kNE}$, the $\kappa B S$ sequence was able to cause a B-cell-specific enhancement of transcriptional stimulation via adjacent NF-kBbinding sites but by itself could not mediate transcriptional activation. In these properties, $\kappa \mathrm{BS}$ is reminiscent of the sequence that binds the high-mobility-group protein-related lymphoid enhancer factor 1 , a protein that bends DNA and potentiates the activity of other factors while being inactive on its own (5).

Nelms and collaborators $(14,15)$ have previously studied nuclear factors binding to the human and mouse Igk enhancers, using probes including the $\kappa B S$ sequences described here. They concluded that the human sequence is a functional octamer-binding site, whereas the corresponding mouse sequence instead binds another nuclear factor referred to as $\kappa \mathrm{BF}-\mathrm{A}$. Our gel shift experiments using mouse and human $\kappa B S$ probes (20a) are consistent with their findings. However, neither the predominantly B-cell-specific Oct-2 protein nor $\kappa \mathrm{BF}-\mathrm{A}$ seems likely to be responsible for the in vivo effect of the $\kappa B S$ element. First, $\kappa B F-A$ can be detected only in stimulated pre-B cells, but not in more mature $B$ cells $(14,20 \mathrm{a})$. Second, comparison of $\kappa \mathrm{BS}$ activity in $\mathrm{v}$-Abl-transformed pre-B cells derived from Oct- $2^{-/-}$mice or their wild-type littermates did not suggest a role for Oct-2 protein in the function of mouse or human $\kappa \mathrm{BS}$ elements (unpublished results in collaboration with Lynn Corcoran). Nevertheless, these studies support an important B-cellspecific regulatory role for the $\mathrm{kBS}$ element and encourage further studies to characterize putative factors with which it interacts.

Our reporter transfection studies indicate that $\mathrm{kNE}$ can eliminate about two-thirds of the transcriptional activation via an adjacent $I g \kappa \kappa B$ site. A functional $\kappa \mathrm{B}$ site is crucial for the transcriptional activity of the Igk intronic enhancer (10), suggesting an important role for $\mathrm{kNE}$ in repressing Igא expression in non-B cells. However, it is presently not possible to estimate the magnitude of the $\mathrm{kNE}$ effect in its natural context in the Igא enhancer. Pierce et al. (16) have previously shown that sequences upstream of the region we have defined can have a strong negative effect in a slightly different experimental context. Thus, it is likely that $\mathrm{\kappa NE}$ is only one element involved in keeping the Igא gene silent until the moment in B-cell development when it is activated.

The sequence homology revealed between $\kappa \mathrm{NE}$ and the B1 family of genomic repeat elements was a surprising finding and may have important implications. A complete B1 element is typically $\sim 140 \mathrm{bp}$ long (23); therefore, the $\mathrm{kNE}$ sequence only represents a part of such an element. Its high homology over $26 \mathrm{bp}$, however, strongly implies that its evolutionary origin is from a B1-like repeat. The presence of a homologous $\kappa \mathrm{NE}$ sequence in the corresponding positions in the human and rabbit Igא J-C intronic enhancers (Fig. 2A) supports the functional significance of $\mathrm{kNE}$ and suggests that this sequence may have evolved from an archaic B1 or Alu-like element and then suffered a deletion some time before these species diverged in evolution.

The idea that B1 elements may have a widespread role in the negative control of gene expression was strongly supported by the finding that a sequence homologous to $\mathrm{kNE}$ and B1 is also shared by another previously identified negative element, namely, TCRSIL2, which is involved in down-regulation of TCR $\alpha$ expression in cells other than $\alpha \beta$-lineage T cells (24). The B1-like sequence in TCRSIL2 is a more typical $\mathrm{B} 1$ element than $\mathrm{kNE}$ is, which suggests a more recent evolutionary origin. Therefore, the ability of the 27-bp $\kappa$ NE- or B1-like TCRSIL2 fragment to negatively regulate reporter gene transcription and to abolish binding of $\mathrm{mB} 1-\mathrm{NF}$ to the $\mathrm{kNE}$ sequence demonstrates that the $\mathrm{B} 1$ homology of the $\mathrm{kNE}$ sequence is functionally relevant. Another 27-bp fragment, corresponding to the most prevalent (consensus) sequence in this region of the B1 elements, however, did not show any negative regulatory activity in our test system and it did not compete with the $\mathrm{kNE}$ probe for mB1-NF binding. Thus, it appears that only certain variants of the B1-like sequences may acquire a negative regulatory function.

A role for some other highly repeated sequences in the negative control of transcription has been suggested by previous studies. The silencer element in the rat insulin 1 locus was identified as a member of a rat long interspersed repetitive sequence family (9). One of the three identified chicken lysozyme silencer elements was found to consist of sequences homologous to the chicken middle repetitive sequence element CR1 (1). Also, although the Alu sequences have not been indicated in negative regulation of any specific genes, their potential for transcriptional repression has been suggested $(20,21)$. 
B1 sequences, as well as many of the other highly repeated genomic sequences, are thought to represent retrotransposons, DNA elements which can be copied into new positions in the genome through RNA intermediates. Evolutionary characterization, as well as rare studies in real time (4), has confirmed that some of repeated sequences, including the Alu elements $(13,22)$, continue to retrotranspose. The notion that these sequences, after perhaps a small number of mutations, can play a physiological role has been an attractive one for many years, but has received little support (recently reviewed by Howard and Sakamoto [8]). Involvement of a mobile DNA element in transcriptional regulation would allow alterations in gene expression to occur with great flexibility, ease, and speed over evolutionary time.

It is tempting to speculate that in addition to Igא and TCR $\alpha$ enhancers, B1-like sequences could be widely used as versatile building blocks that confer a negative transcriptional effect onto a variety of different cell type- and differentiation-specific enhancers. A large number of the murine and primate genes so far sequenced and characterized contain B1- and $A l u$-like sequences in positions which could imply regulatory significance (20a). Further studies addressing the detailed sequence and spatial requirements functionally critical for B1-like negative elements should help predicting which ones are likely to be involved in the control of gene expression.

\section{ACKNOWLEDGMENTS}

We thank Chris Roman for valuable comments on the manuscript and Patricia Cortes for help and suggestions in the band shift experiments.

K.S. was supported by an EMBO postdoctoral fellowship. This work was supported by NIH grant GM39458-04 to D.B.

\section{REFERENCES}

1. Baniahmad, A., M. Muller, C. Steiner, and R. Renkawitz. 1987. Activity of two different silencer elements of the chicken lysozyme gene can be compensated by enhancer elements. EMBO J. 6:2297-2303.

2. Bauerle, P. A., and D. Baltimore. 1988. Activation of DNAbinding activity in an apparently cytoplasmic precursor of the NF-кB transcription factor. Cell 53:211-217.

3. Dignam, J. D., R. M. Lebovitz, and R. G. Roeder. 1983. Accurate transcription initiation by RNA polymerase II in a soluble extract from isolated mammalian nuclei. Nucleic Acids Res. 11:1475-1489.

4. Dombroski, B. A., S. L. Mathias, E. Nanthakumar, A. F. Scott, and H. H. Kazazian, Jr. 1991. Isolation of an active human transposable element. Nature (London) 254:1805-1808.

5. Giese, K., J. Cox, and R. Grosschedl. 1992. The HMG domain of lymphoid enhancer factor 1 bends DNA and facilitates assembly of functional nucleoprotein structures. Cell 69:1-20.

6. Gilman, M. Z., R. N. Wilson, and R. A. Weinberg. 1986. Multiple protein-binding sites in the 5 '-flanking region regulate c-fos expression. Mol. Cell. Biol. 6:4305-4316.

7. Grosschedl, R., and D. Baltimore. 1985 . Cell type specificity of immunoglobin gene expression is regulated by at least 3 DNA sequence elements. Cell 41:885-897.

8. Howard, B. H., and K. Sakamoto. 1990. Alu interspersed repeats: selfish DNA or a functional gene family. New Biol. 2:759-770.

9. Laimins, L., M. Holmgren-Konig, and G. Khoury. 1986. Transcriptional "silencer" element in rat repetitive sequences associated with the rat insulin 1 locus. Proc. Natl. Acad. Sci. USA 83:3151-3155.

10. Lenardo, M., J. W. Pierce, and D. Baltimore. 1987. Proteinbinding sites in Ig gene enhancers determine transcriptional activity and inducibility. Science 236:1573-1577.

11. Libermann, T. A., and D. Baltimore. 1991. Transcriptional regulation of immunoglobulin gene expression, p. 385-406. In P. Cohen and J. G. Foulkes (ed.), The hormonal control regulation of gene transcription. Elsevier Science Publishers B.V. (Biomedical Division), Amsterdam.

12. Maraia, R. J. 1991. The subset of mouse B1 (Alu-equivalent) sequences expressed as small processed cytoplasmic tran scripts. Nucleic Acids Res. 19:5695-5702.

13. Muratani, K., T. Hada, Y. Yamamoto, T. Kaneko, Y. Shigeto, T. Ohue, J. Furuyama, and K. Higashino. 1991. Inactivation of the cholinesterase gene by $A l u$ insertion: possible mechanism for human gene transposition. Proc. Natl. Acad. Sci. USA 88: 11315-11319.

14. Nelms, K., R. Hromas, and B. Van Ness. 1990. Identification of a second inducible DNA-protein interaction in the kappa immunoglobulin enhancer. Nucleic Acids Res. 18:1037-1043.

15. Nelms, K., and B. Van Ness. 1990. Identification of an octamerbinding in the human kappa light-chain enhancer. Mol. Cell. Biol. 10:3843-3846.

16. Pierce, J. W., A. M. Gifford, and D. Baltimore. 1991. Silencing of the expression of the immunoglobulin kappa gene in non-B cells. Mol. Cell. Biol. 11:1431-1437.

17. Pierce, J. W., M. Lenardo, and D. Baltimore. 1988. Oligonucleotide that binds nuclear factor NF- $\mathrm{KB}$ acts as a lymphoidspecific and inducible enhancer element. Proc. Natl. Acad. Sci. USA 85:1482-1486.

18. Quentin, Y. 1989. Successive waves of fixation of B1 variants in rodent lineage history. J. Mol. Evol. 28:299-305.

19. Roesler, W. J., G. R. Vanderbark, and R. W. Hanson. 1988 Cyclic AMP and the induction of eukaryotic gene transcription. J. Biol. Chem. 263:9063-9066.

20. Saffer, J. D., and S. J. Thurston. 1989. A negative regulatory element with properties similar to those of enhancers is contained within an Alu sequence. Mol. Cell. Biol. 9:355-364.

20a.Saksela, K., and D. Baltimore. Unpublished data.

21. Tomilin, N. V., S. M. M. Iguchi-Ariga, and H. Ariga. 1990. Transcription and replication silencer element is present within conserved region of human Alu repeats interacting with nuclear protein. FEBS Lett. 263:69-72.

22. Wallace, M. R., L. B. Andersen, A. M. Saulino, P. E. Gregory, T. W. Glover, and F. S. Collins. 1991. A de novo Alu insertion results in neurofibromatosis type 1. Nature (London) 353:864 866.

23. Weiner, A. M., P. L. Deininger, and A. Efstratiadis. 1986. Nonviral retroposons: genes, pseudogenes, and transposable elements generated by the reverse flow of genetic information. Annu. Rev. Biochem. 55:631-661.

24. Winoto, A., and D. Baltimore. 1989. $\alpha \beta$ lineage-specific expression of the $\alpha \mathrm{T}$ cell receptor gene by nearby silencers. Cell 59:649-655. 